\title{
Existence of Dual Equations by Means of Strong Necessary Conditions - Analysis of Integrability of Partial Differential Nonlinear Equations
}

\author{
KSOKALSKI ${ }^{\dagger}, T$ WIETECHA ${ }^{\ddagger}$ and D SOKALSKA ${ }^{\dagger \ddagger}$ \\ $\dagger$ Institute of Computer Science, Technical University of Czȩstochowa, \\ Al.Armii Krajowej 17, 42-200 Czȩstochowa, Poland \\ E-mail: sokalski@el.pcz.czest.pl \\ $\ddagger$ Institute of Physics, Jagellonian University, \\ 30-059 Kraków, ul.Reymonta 4, Poland \\ E-mail: wietecha@izis.if.uj.edu.pl \\ †Institute of Physics, Technical University of Cracow, \\ 30-084 Kraków, ul. Podchorąizych 1, Poland
}

Received November 05, 2003; Accepted October 07, 2004

\begin{abstract}
A concept of strong necessary conditions for extremum of functional has been applied for analysis an existence of dual equations for a system of two nonlinear Partial Differential Equations (PDE) in 1+1 dimensions. We consider two types of the dual equations: the Bäcklund transformations and the Bogomolny equations. A general form of the second order PDE with a derivative-less non-linear term has been considered. In the case of a coupled system of equations the general conditions for the existence of the Bogomolny decomposition are derived. In the case of an uncoupled system of equations the Bogomolny equations become the Bäcklund transformations. It has been found a denumerable classes of coupled systems possessing the Bogomolny relationship. Weaken the method into semi-strong necessary conditions is presented together with an application to the Lax hierarchy. The method basing on both the strong and the semi-strong necessary condition concept reduces the derivation of the dual equations to an algorithm.
\end{abstract}

\section{Introduction}

Integrable nonlinear partial differential equations have attracted much interest both in physics and in mathematics. These equations have rich mathematical structures which showed up behind them. Properties of such structures are: Existence of the Lax pairs, the Bäcklund transformations, the Bogomolny relationship, infinitely many local conservation laws, the Miura maps and the (bi-)Hamiltonian structures [1], [2], [3]. In this paper we present a new way of derivation of dual equations (the Bäcklund transformations, the 
Bogomolny equations and others) for nonlinear partial differential evolution equations. There are different ways in which the dual equations may be achieved. For the derivation of the Bäcklund transformations we refer to [4] - [7] as well as to the Appendix A, and for the derivation of the Bogomolny equations to [8]-[14] as well as to the Appendix B. Recently, a new variational approach to the Bäcklund transformations [15] and to the Bogomolny decomposition [16] has been introduced on the basis of the strong necessary condition concept [17] - [20]. The concept is grounded on the assumption that the considered equation results from the necessary condition for an extremum of a functional to exist:

$$
\delta \Phi[u]=0,
$$

where:

$$
\begin{aligned}
& \Phi[u]=\int_{E^{2}} F\left(u, u_{, x}, u_{, t}\right) d x d t \\
& \delta \Phi[u]=\int_{E^{2}}\left(F_{, u} \delta u+F_{, u_{, x}} \delta u_{, x}+F_{, u, t} \delta u_{, t}\right) d x d t
\end{aligned}
$$

and

$$
\begin{array}{r}
u(x, t) \in C^{2}(\mathbf{E} \times \mathbf{E}) \\
F\left(u, u_{, x}, u_{, t}\right) \in C^{2}\left(\mathbf{R}^{3}\right)
\end{array}
$$

Since we relate the developed method to the classical field theory we assume that the structure of the Euclidean space for a domain of the independent variables $x, t$. Both variables $x$ and $t$ are equivalent. Therefore, we have to determine the asymptotic conditions for the dependent variables:

$$
\lim _{\substack{+x \rightarrow-\infty}} u(x, t)=\lim _{\substack{+t \rightarrow-\infty}} u(x, t)=\alpha
$$

where $\alpha$ is an arbitrary constant $(|\alpha|<\infty)$. For $u_{, x}(x, t)$ and $u_{, t}(x, t)$ we assume analogous conditions. These conditions lead to the Euler-Lagrange equation:

$$
F_{, u}-D_{x} F_{, u, x}-D_{t} F_{, u, t}=0
$$

However, the (1.1) can be satisfied by assuming the strong necessary conditions:

$$
\begin{aligned}
& F_{, u, x}=0 \\
& F_{, u, t}=0 \\
& F_{, u}=0 .
\end{aligned}
$$

Note that all solutions of (1.6),(1.7) and (1.8) satisfy (1.5). However, in most cases the set of solutions of $(1.6),(1.7)$ and $(1.8)$ is trivial $(u=c o n s t)$ or empty. In order to extend this set to a nontrivial one we use the gauge transformation of (1.2)

$$
\Phi \rightarrow \Phi+I
$$

and instead of (1.5) we apply (1.6),(1.7) and (1.8). The scaling functional $I$ is invariant with respect to the local variation of $u(x, t): \delta I \equiv 0$. Therefore, the Euler-Lagrange 
equations resulting from the extremum of $\Phi$ and the extremum of $\Phi+I$ are equivalent. The (1.6),(1.7) and (1.8) are not invariant however, with respect to $\Phi \rightarrow \Phi+I$, i.e. the gauge transformation contributes to the strong necessary condition. This contribution can extend the subset of solutions to the nontrivial one. In such a way, we derive simpler differential equations for extremales of $\Phi$, solutions of which form a subset of solutions of the Euler-Lagrange equation. The aim of this paper is to present the application of the strong necessary conditions to the analysis of the integrability of nonlinear partial differential evolution equations. In the case of the integrable Euler-Lagrange equation the concept of strong necessary conditions is applied to a derivation of the Bäcklund transformations and in the case of two coupled equations to a derivation of the Bogomolny equations. The paper is organized as follows: in Section 2 we apply the concept of the strong necessary conditions to the non-linear PDE in $1+1$ dimensions of the second order with the derivative-less non-linear term. We derive the Bäcklund transformations and the integrability conditions. Section 3 presents the same investigation program applied to the system of two coupled PDE equations in $1+1$ dimensions. In Section 4 we extend our method into a semi-strong necessary conditions concept. As an application of this concept we derive the auto-Bäcklund transformations for the fifth-order KdV equation. In the summary section we discuss the algorithmic structure of the presented method and differences between the Bäcklund transformations and the Bogomolny equations.

\section{Dual equations for decoupled second order PDE in $1+1$ dimensions}

Let us analyze an integrability of the following class of equations:

$$
a u_{, x x}+b u_{, t t}+2 c u_{, x t}=P(u)
$$

In order to apply the strong necessary conditions concept we supplemented the (2.1) with an independent similar equation and we have:

$$
\begin{aligned}
a u_{, x x}+b u_{, t t}+2 c u_{, x t} & =P(u) \\
\tilde{a} v_{, x x}+\tilde{b} v_{, t t}+2 \tilde{c} v_{, x t} & =Q(v)
\end{aligned}
$$

where:

$$
u(x, t) \in C^{2}(\mathbf{E} \times \mathbf{E}), \quad v(x, t) \in C^{2}(\mathbf{E} \times \mathbf{E}),
$$

and $P(u) \in L^{1}(\mathbf{R}), Q(v) \in L^{1}(\mathbf{R})$. These conditions guarantee the existence of $p(u)$ and $q(v)$ such that: $p_{, u}=P(u), q_{, v}=Q(v)$. The equation parameters $a, b, c, \tilde{a}, \tilde{b}, \tilde{c}$ are arbitrary real constants and $\lambda$ is the Lagrangean multiplier. The $u$ and $v$ satisfy the asymptotic conditions (1.4) which associates (2.2) with the $\pi_{2}\left(S^{2}\right)$ homotopy group. Let us formulate the following problem: What are the forms of the $P(u)$ and the $Q(v)$ and what is the subspace of the equation parameters for which the (2.2) possesses the Bäcklund transformation? The strong necessary conditions start from the generating functional:

$$
\begin{aligned}
\Phi[u, v]= & \int_{E^{2}}\left[\frac{a}{2} u_{, x}^{2}+\frac{b}{2} u_{, t}^{2}+c u_{, x} u, t+p(u)+\right. \\
& \left.\lambda\left(\frac{\tilde{a}}{2} v_{, x}^{2}+\frac{\tilde{b}}{2} v_{, t}^{2}+\tilde{c} v_{, x} v_{, t}+q(v)\right)\right] d x d t
\end{aligned}
$$




\subsection{The topological invariant}

Crucial role in the concept of the strong necessary conditions plays the topological invariant $I$. It must be defined on the same domain as $\Phi[u, v]$ :

$$
I[u, v]=\int_{E^{2}} J\left(u, v, u_{, x}, v_{, x}, u_{, t}, v_{, t}\right) d x d t
$$

where $J\left(u, v, u_{, x}, v_{, x}, u_{, t}, v_{, t}\right) \in C^{2}\left(\mathbf{R}^{6}\right)$. From the definition of the topological invariant we derive the following conditions for $J$ :

$$
\begin{aligned}
\hat{\mathcal{L}}^{u} J\left(u, v, u_{, x}, v_{, x}, u_{, t}, v_{, t}\right) & \equiv 0 \\
\hat{\mathcal{L}}^{v} J\left(u, v, u_{, x}, v_{, x}, u_{, t}, v_{, t}\right) & \equiv 0
\end{aligned}
$$

where

$$
\begin{aligned}
\hat{\mathcal{L}}^{u} & =\frac{\partial}{\partial u}-D_{x} \frac{\partial}{\partial u_{, x}}-D_{t} \frac{\partial}{\partial u_{, t}} \\
\hat{\mathcal{L}}^{v} & =\frac{\partial}{\partial v}-D_{x} \frac{\partial}{\partial v_{, x}}-D_{t} \frac{\partial}{\partial v_{, t}}
\end{aligned}
$$

We can formally treat (2.6) and (2.7) as a set of the simultaneous partial differential equations for $J\left(u, v, u_{, x}, v_{, x}, u_{, t}, v_{, t}\right)$. General solution of (2.6), (2.7) leads to the general form for (2.5):

$$
I[u, v]=I_{1}+I_{2}+I_{3}
$$

where is $\left\{I_{1}, I_{2}, I_{3}\right\}$ complete set of invariants.

Theorem 1. [22].

Let $H_{J}=\left\{J: J \in C^{2}\left(\mathbf{R}^{6}\right),\left(u, v, u_{, x}, v_{, x}, u_{, t}, v_{, t}\right) \stackrel{J}{\longrightarrow} J\left(u, v, u_{, x}, v_{, x}, u_{, t}, v_{, t}\right) \in \mathbf{R}\right\}$ be the linear space. Then the set of invariants

$$
\begin{array}{r}
I_{1}=\int_{E^{2}} J_{1} d x d t=\int_{E^{2}} G_{1}(u, v)\left(u_{, x} v_{, t}-u_{, t} v_{, x}\right) d x d t \\
I_{2}=\int_{E^{2}} J_{2} d x d t=\int_{E^{2}} D_{x} G_{2}(u, v) d x d t \\
I_{3}=\int_{E^{2}} J_{2} d x d t=\int_{E^{2}} D_{t} G_{3}(u, v) d x d t
\end{array}
$$

where $G_{1}(u, v) \in C^{2}(\mathbf{R} \times \mathbf{R}), G_{2}(u, v), G_{3}(u, v) \in C^{3}(\mathbf{R} \times \mathbf{R})$, is complete in the sense that $J_{1}, J_{2}, J_{3}$ is a complete base of ker $\hat{\mathcal{L}}$.

Proof. By the assumption $J\left(u, v, u_{, x}, v_{, x}, u_{, t}, v_{, t}\right)$ can not depend on the second derivatives of $u$ and $v$. Let us derive conditions for $J$ resulting from this assumption. Expanding (2.6), 
(2.7) we obtain:

$$
\begin{array}{r}
\frac{\partial}{\partial u} J-\left(\frac{\partial^{2}}{\partial u \partial u_{, x}} J\right) u_{, x}-\left(\frac{\partial^{2}}{\partial u_{, x} \partial v} J\right) v_{, x}-\left(\frac{\partial^{2}}{\partial u_{, x}^{2}} J\right) u_{, x x}- \\
\left(\frac{\partial^{2}}{\partial u_{, x} \partial v_{, x}} J\right) v_{, x x}-2\left(\frac{\partial^{2}}{\partial u_{, t} \partial u_{, x}} J\right) u_{, x t}-\left(\frac{\partial^{2}}{\partial u_{, x} \partial v_{, t}} J\right) v_{, x t}-\left(\frac{\partial^{2}}{\partial u \partial u_{, t}} J\right) u_{, t}- \\
\left(\frac{\partial^{2}}{\partial u_{, t} \partial v} J\right) v_{, t}-\left(\frac{\partial^{2}}{\partial u_{, t} \partial v_{, x}} J\right) v_{, x t}-\left(\frac{\partial^{2}}{\partial u_{, t}^{2}} J\right) u_{, t t}-\left(\frac{\partial^{2}}{\partial u_{, t} \partial v_{, t}} J\right) v_{, t t} \equiv 0 \\
\frac{\partial}{\partial v} J-\left(\frac{\partial^{2}}{\partial u \partial v_{, x}} J\right) u_{, x}-\left(\frac{\partial^{2}}{\partial v \partial v_{, x}} J\right) v_{, x}-\left(\frac{\partial^{2}}{\partial u_{, x} \partial v_{, x}} J\right) u_{, x x}- \\
\left(\frac{\partial^{2}}{\partial v_{, x}^{2}} J\right) v_{, x x}-\left(\frac{\partial^{2}}{\partial u_{, t} \partial v_{, x}} J\right) u_{, x t}-2\left(\frac{\partial^{2}}{\partial v_{, t} \partial v_{, x}} J\right) v_{, x t}-\left(\frac{\partial^{2}}{\partial u \partial v_{, t}} J\right) u_{, t}- \\
\left(\frac{\partial^{2}}{\partial v \partial v_{, t}} J\right) v_{, t}-\left(\frac{\partial^{2}}{\partial u_{, x} \partial v_{, t}} J\right) u_{, x t}-\left(\frac{\partial^{2}}{\partial u_{, t} \partial v_{, t}} J\right) u_{, t t}-\left(\frac{\partial^{2}}{\partial v_{, t}^{2}} J\right) v_{, t t} \equiv 0
\end{array}
$$

Therefore, all function coefficients of the second derivatives $u_{, x x}, u_{, x t}, u_{, t t}, v_{, x x}, v_{, x t}, v_{, t t}$ in (2.14), (2.15) must vanish:

$$
\begin{array}{r}
\frac{\partial^{2}}{\partial u_{, x}^{2}} J \equiv 0, \quad \frac{\partial^{2}}{\partial u_{, x} \partial v_{, x}} J \equiv 0, \quad \frac{\partial^{2}}{\partial u_{, t} \partial u_{, x}} J \equiv 0, \\
\frac{\partial^{2}}{\partial u_{, x} \partial v_{, t}} J+\frac{\partial^{2}}{\partial u_{, t} \partial v_{, x}} J \equiv 0, \quad \frac{\partial^{2}}{\partial u_{, t}^{2}} J \equiv 0, \quad \frac{\partial^{2}}{\partial u_{, t} \partial v_{, t}} J \equiv 0, \\
\frac{\partial^{2}}{\partial v_{, x}^{2}} J \equiv 0, \quad \frac{\partial^{2}}{\partial v_{, t} \partial v_{, x}} J \equiv 0, \frac{\partial^{2}}{\partial v_{, t}^{2}} J \equiv 0
\end{array}
$$

These constrains lead to the following form for $J\left(u, v, u_{, x}, v_{, x}, u_{, t}, v_{, t}\right)$ :

$$
J=G_{1}(u, v)\left(u_{, x} v_{, t}-u_{, t} v_{, x}\right)+A u_{, x}+B u_{, t}+C v_{, x}+D v_{, t}+E
$$

where, $A(u, v), B(u, v), C(u, v), D(u, v), E(u, v) \in C^{2}(\mathbf{R} \times \mathbf{R})$. Substituting (2.17) to (2.14), (2.15) we derive additional constrains for $A(u, v), B(u, v), C(u, v), D(u, v), E(u, v)$ :

$$
\begin{aligned}
\frac{\partial E(u, v)}{\partial u} \equiv 0, \quad \frac{\partial E(u, v)}{\partial v} & \equiv 0 \\
\frac{\partial C(u, v)}{\partial u}-\frac{\partial A(u, v)}{\partial v} & \equiv 0 \\
\frac{\partial D(u, v)}{\partial u}-\frac{\partial B(u, v)}{\partial v} & \equiv 0
\end{aligned}
$$

Therefore

$$
\begin{array}{r}
E(u, v) \equiv \text { const } \\
\exists G_{2}(u, v): A(u, v)=\frac{\partial G_{2}(u, v)}{\partial u} \text { and } C(u, v)=\frac{\partial G_{2}(u, v)}{\partial v} \\
\exists G_{3}(u, v): B(u, v)=\frac{\partial G_{3}(u, v)}{\partial u} \text { and } D(u, v)=\frac{\partial G_{3}(u, v)}{\partial v}
\end{array}
$$




\subsection{The strong necessary conditions}

Theorem 2. The Euler equations for (2.4) are invariant with respect to the gauge transformation of $\Phi[u, v]$ :

$$
\Phi^{*}=\Phi+I_{1}+I_{2}+I_{3}
$$

Proof. Since $\delta I \equiv 0$, the variations of the both sides of (2.24) lead to identical Euler's equations for $\Phi^{*}$ and $\Phi: \delta \Phi^{*}[u, v]=\delta \Phi[u, v]=0$

However, as we have mentioned in the Introduction, the strong necessary conditions (1.6)(1.8) are not invariant with respect to (2.24):

$$
\begin{aligned}
& u: \quad P(u)+G_{1, u}\left(u_{, x} v_{, t}-u_{, t} v_{, x}\right)+G_{2, u u} u_{, x}+G_{2, u v} v_{, x}+ \\
& G_{3, u u} u_{, t}+G_{3, u v} v_{, t}=0 \\
& v: \quad \lambda Q(v)+G_{1, v}\left(u_{, x} v_{, t}-u_{, t} v_{, x}\right)+G_{2, u v} u_{, x}+G_{2, v v} v_{, x}+ \\
& G_{3, v u} u_{, t}+G_{3, v v} v_{, t}=0 \\
& u_{, x}: \quad a u_{, x}+c u_{, t}+G_{1} v_{, t}+G_{2, u}=0 \\
& u_{, t}: \quad b u_{, t}+c u_{, x}-G_{1} v_{, x}+G_{3, u}=0 \\
& v_{, x}: \quad \lambda \tilde{a} v_{, x}+\lambda \tilde{c} v_{, t}-G_{1} u_{, t}+G_{2, v}=0 \\
& v_{, t}: \quad \lambda \tilde{b} v_{, t}+\lambda \tilde{c} v_{, x}+G_{1} u_{, x}+G_{3, v}=0
\end{aligned}
$$

This property makes a chance to derive nontrivial solutions of (2.2) from (2.25)-(2.30). However, since the necessary conditions (2.25)-(2.30) are stronger then (2.2) in general case the solution space of (2.25)-(2.30) is a subspace of the solution space of (2.2). The Eqs. $(2.25) \div(2.30)$ have to be self-consistent. Note that $(2.27)-(2.30)$ characterize all $1+1-$ dimensional PDE of the type (2.1) because they do not depend on the $P(u)$ (since $Q(v)$ is optional we do not mention it in this place). Formally, we have six simultaneous equations for the five unknown functions: $u, v, G_{1}, G_{2}, G_{3}$. The system (2.25)-(2.30) becomes the Bäcklund transformations if there exists such an Ansatz for the $G_{1}, G_{2}, G_{3}$ for which the above equations reduce to two equations for the $u$ and $v[15],[20]$. Essential role in this procedure plays reduction of the number of independent equations by an appropriate choice of the $G_{1}, G_{2}, G_{3}$. Only for very special $P(u)$ and $Q(v)$ such an Ansatz exists [23]. In the most cases of $P$ and $Q$ the system (2.25)-(2.30) cannot be reduced to the Bäcklund transformations. The (2.25), (2.26) and (2.27)-(2.30) require different ways of treatment. In order to make all $(2.25),(2.26)$ and $(2.27)-(2.30)$ self-consistent we reduce the (2.25), (2.26) to a tautology and make the (2.27)-(2.30) linear dependent.

\subsection{Reduction of the (2.25)-(2.30) to the Bäcklund transformations}

We will reduce the system (2.27)-(2.30) to two equations by setting the following condition:

$$
\operatorname{rank}\left[\begin{array}{ccccc}
a & c & 0 & G_{1} & G_{2, u} \\
c & b & -G_{1} & 0 & G_{3, u} \\
0 & -G_{1} & \lambda \tilde{a} & \lambda \tilde{c} & G_{2, v} \\
G_{1} & 0 & \lambda \tilde{c} & \lambda \tilde{b} & G_{3, v}
\end{array}\right]=2
$$


Then using (2.27)-(2.30) and the constrains for the equation parameters and $G_{i}$ functions resulting from $(2.31)$, we reduce $(2.25),(2.26)$ to the tautology. The $(2.31)$ can be satisfied by three different classes of the equation parameters and the $G_{i}$ functions.

\subsubsection{The class: $G_{1} \neq 0, a \neq 0, \tilde{a} \neq 0$}

The procedure reducing (2.25)-(2.30) to the Bäcklund transformations we close in four points.

I. Transforming the matrix of equations (2.27)-(2.30) to the Gauss-Jordan form we satisfy (2.31) by setting the following relations for the equation parameters and $G_{1}$ :

$$
\begin{aligned}
& \tilde{c}=c \frac{\tilde{a}}{a} \\
& \tilde{b}=b \frac{\tilde{a}}{a} \\
& G_{1}^{2}=\frac{\lambda \tilde{a}}{a}\left(a b-c^{2}\right)
\end{aligned}
$$

as well as

II. the following set of differential equations for $G_{2}$ and $G_{3}$ :

$$
\begin{aligned}
\left(a b-c^{2}\right) G_{2, v}+G_{1}\left(a G_{3, u}-c G_{2, u}\right) & =0 \\
a G_{3, v}-G_{1} G_{2, u}-c G_{2, v} & =0
\end{aligned}
$$

where

$$
G_{1} \neq 0, a \neq 0, \tilde{a} \neq 0
$$

It results from (2.36) and (2.33) that

$$
\operatorname{Det}\left[\begin{array}{ll}
a & c \\
c & b
\end{array}\right] \neq 0
$$

and $G_{1}=$ const. Therefore, we reduce four equations (2.27)-(2.30) to the Bäcklund transformations represented by (2.27)-(2.28).

III. Now we will make $(2.25),(2.26)$ a tautology. Taking into account (2.33) we solve (2.27) and (2.28) with respect to $u_{, x}$ and $u_{, t}$ and substitute them to (2.25),(2.26) and obtain:

$$
\begin{array}{r}
P(u)\left(a b-c^{2}\right)+\mathcal{A}_{1} v_{, x}+\mathcal{B}_{1} v_{, t}+\mathcal{C}_{1}=0 \\
G_{1}^{2} a Q(v)+\mathcal{A}_{2} v_{, x}+\mathcal{B}_{2} v_{, t}+\mathcal{C}_{2}=0
\end{array}
$$

where

$$
\begin{array}{r}
\mathcal{A}_{1}=-c G_{1} G_{2, u u}+a b G_{2, u v}-c^{2} G_{2, u v}+a G_{1} G_{3, u u} \\
\mathcal{B}_{1}=-b G_{1} G_{2, u u}+c G_{1} G_{3, u u}+a b G_{3, u v}-c^{2} G_{3, u v} \\
\mathcal{C}_{1}=c G_{2, u u} G_{3, u}+c G_{3, u u} G_{2, u}-a G_{3, u u} G_{3, u}-b G_{2, u u} G_{2, u} \\
\mathcal{A}_{2}=-\tilde{a} c G_{1} G_{2, u v}+\tilde{a} a b G_{2, v v}-\tilde{a} c^{2} G_{2, v v}+\tilde{a} a G_{1} G_{3, u v} \\
\mathcal{B}_{2}=-\tilde{a} b G_{1} G_{2, u v}+\tilde{a} c G_{1} G_{3, u v}+\tilde{a} a b G_{3, v v}-\tilde{a} c^{2} G_{3, v v} \\
\mathcal{C}_{2}=\tilde{a} c G_{2, u v} G_{3, u}+\tilde{a} c G_{3, u v} G_{2, u}-\tilde{a} a G_{3, u v} G_{3, u}-\tilde{a} b G_{2, u v} G_{2, u}
\end{array}
$$


Theorem 3. If (2.34) and (2.35) is true then

$$
\mathcal{A}_{1} \equiv 0, \quad \mathcal{B}_{1} \equiv 0, \quad \mathcal{A}_{2} \equiv 0, \quad \mathcal{B}_{2} \equiv 0
$$

Proof. Inserting (2.34) and (2.35) to (2.40),(2.41),(2.43),(2.44) we obtain (2.46).

Finally, (2.25),(2.26) become:

$$
\begin{gathered}
P(u)\left(a b-c^{2}\right)+\frac{1}{2}\left(-a G_{3, u}^{2}-b G_{2, u}^{2}+2 c G_{2, u} G_{3, u}\right)_{, u}=0 \\
\lambda Q(v)\left(a b-c^{2}\right)+\frac{1}{2}\left(-a G_{3, u}^{2}-b G_{2, u}^{2}+2 c G_{2, u} G_{3, u}\right)_{, v}=0
\end{gathered}
$$

(2.47) and (2.48) can be integrated into one condition:

$$
2\left(a b-c^{2}\right)(p(u)+\lambda q(v))+\left(-a G_{3, u}^{2}-b G_{2, u}^{2}+2 c G_{2, u} G_{3, u}\right)=C^{\prime}
$$

where $C^{\prime} \in \mathbf{R}$.

Solving (2.34), (2.35) we obtain solutions for $G_{2}$ and $G_{3}$ :

$$
\begin{aligned}
G_{2} & =\frac{\left(-\sqrt{-a b+c^{2}}+c\right) F_{2}(\zeta)}{b}+\frac{\left(\sqrt{-a b+c^{2}}+c\right) F_{1}(\xi)}{b}+C_{1}, \\
G_{3} & =F_{2}(\zeta)+F_{1}(\xi)
\end{aligned}
$$

where

$$
\begin{aligned}
& \xi=-1 / 2 v \frac{1}{\sqrt{-\frac{a b-c^{2}}{G_{1}^{2}}}}+1 / 2 u, \\
& \zeta=v+\sqrt{-\frac{a b-c^{2}}{G_{1}^{2}}} u,
\end{aligned}
$$

$F_{1}, F_{2}$ are arbitrary functions of $C^{2}$. In order to determine admissible set of solutions of (2.47) and (2.48) we set the following conditions: $\frac{\partial}{\partial v} P(u)=0$ and $\frac{\partial}{\partial u} Q(v)=0$, which can be expressed by the $F_{1}$ and $F_{2}$ :

$$
4 \mathrm{D}\left(F_{1}\right)(\xi)\left(D^{(3)}\right)\left(F_{2}\right)(\zeta)\left(a b-c^{2}\right)+\left(D^{(3)}\right)\left(F_{1}\right)(\xi) G_{1}^{2} \mathrm{D}\left(F_{2}\right)(\zeta)=0
$$

where

$$
D^{(p)}(F)(\zeta)=\frac{d^{p}}{d \zeta^{p}} F(\zeta)
$$

Separating variables in (2.54) we obtain:

$$
\frac{F_{1}^{\prime \prime \prime}(\xi)}{F_{1}^{\prime}(\xi)}=-\frac{4\left(a b-c^{2}\right)}{G_{1}^{2}} \frac{F_{2}^{\prime \prime \prime}(\zeta)}{F_{2}^{\prime}(\zeta)}=\omega
$$

where $\omega$ is a separation constant.

IV. Therefore, if the equation coefficients satisfy (2.32), if $F_{1}$ and $F_{2}$ satisfy (2.56) then 
(2.49) is the tautology and the equations (2.27) and (2.28) are the Bäcklund transformations for the system (2.2) subjected to (2.32). The solutions of (2.56) together with (2.50), (2.51) and (2.47), (2.48) determine all subjected to (2.32) systems possessing the Bäcklund transformations of the following forms:

$$
\begin{aligned}
& a u_{, x}+c u_{, t}+G_{1} v_{, t}+G_{2, u}=0 \\
& b u_{, t}+c u_{, x}-G_{1} v_{, x}+G_{3, u}=0
\end{aligned}
$$

where $G_{2}$ and $G_{3}$ are given by (2.50), (2.51) and (2.52), (2.53). In the particular case of $a=\tilde{a}=b=\tilde{b}=1$ and $c=\tilde{c}=0$ we derive the known result for the non-linear wave equations:

$$
\begin{array}{r}
G_{1}= \pm 1, \quad \lambda=1 \\
G_{2}=-i F_{2}(v+i u)+i F_{1}\left(\frac{u+i v}{2}\right) \\
G_{3}=F_{2}(v+i u)+F_{1}\left(\frac{u+i v}{2}\right) \\
p(u)+q(v)=F_{1}^{\prime} F_{2}^{\prime} \\
u_{, x}+v_{, t}+\frac{i}{2} F_{1}^{\prime}+F_{2}^{\prime}=0 \\
u_{, t}-v_{, x}+\frac{1}{2} F_{1}^{\prime}+i F_{2}^{\prime}=0
\end{array}
$$

where $F_{1}, F_{2}$ satisfy (2.56). Therefore we have obtained results for the elliptic equations. This means that the results of this subsection concern whole class of the elliptic equations covering their particular form of the Klein-Gordon type [20], [24] - [28].

\subsubsection{The class: $G_{1} \neq 0, a=0, \tilde{a}=0$}

Note that $\tilde{a}=0$ results from $a=0$. Repeating whole procedure of the previous subsection we derive the following results.

I. The conditions for the equation parameters and $G_{1}$ :

$$
\begin{aligned}
\tilde{c} b & =c \tilde{b} \\
G_{1}^{2} & =-\lambda c \tilde{c}
\end{aligned}
$$

In order to much the parameter conditions of this class we have to set also the following conditions: $c \neq 0$ and $\tilde{c} \neq 0$.

II. The following set of the differential equations for $G_{2}$ and $G_{3}$ :

$$
\begin{aligned}
c G_{2, v}+G_{1} G_{2, u} & =0 \\
c^{2} G_{3, v}-c G_{1} G_{3, u}+b G_{1} G_{2, u} & =0
\end{aligned}
$$

III. The following condition for $p(u)$ and $q(v)$ :

$$
\frac{b}{2} G_{2, u}{ }^{2}-c G_{2, u} G_{3, u}+c^{2}(p(u)+\lambda q(v))=C^{\prime \prime}
$$


where $C^{\prime \prime} \in \mathbf{R}$.

Solving (2.62), (2.63) we obtain solutions for $G_{2}$ and $G_{3}$ :

$$
\begin{array}{r}
G_{2}(u, v)=F_{1}(x), \\
G_{3}(u, v)=\frac{1}{2} \frac{b F_{1}(x)+2 F_{2}(y) c}{c}
\end{array}
$$

where

$$
\begin{array}{r}
x=-\frac{-v G_{1}+c u}{G_{1}}, \\
y=\frac{v G_{1}+c u}{G_{1}}
\end{array}
$$

and $F_{1}, F_{2}$ are arbitrary functions of $C^{2}$. Expressing $P(u)$ and $Q(v)$ by $F_{1}$ and $F_{2}$ we obtain:

$$
\begin{gathered}
P(u)=\frac{c^{2}}{G_{1}^{3}}\left(\left(D^{(2)}\right)\left(F_{1}\right)(x) \mathrm{D}\left(F_{2}\right)(y)-\mathrm{D}\left(F_{1}\right)(x)\left(D^{(2)}\right)\left(F_{2}\right)(y)\right), \\
Q(v)=-\frac{c}{\lambda G_{1}^{2}}\left(\left(D^{(2)}\right)\left(F_{1}\right)(x) \mathrm{D}\left(F_{2}\right)(y)+\mathrm{D}\left(F_{1}\right)(x)\left(D^{(2)}\right)\left(F_{2}\right)(y)\right),
\end{gathered}
$$

In order to determine admissible set of solutions of (2.69) and (2.70) we set the following conditions: $\frac{\partial}{\partial v} P(u)=0$ and $\frac{\partial}{\partial u} Q(v)=0$, which can be expressed by the $F_{1}$ and $F_{2}$ :

$$
\frac{F_{1}^{\prime \prime \prime}(x)}{F_{1}^{\prime}(x)}=\frac{F_{2}^{\prime \prime \prime}(y)}{F_{2}^{\prime}(y)}=\omega
$$

The separation constant $\omega$ labels the solutions of the one parameter family equivalent to the results tabulated in [20].

IV. And the Bäcklund transformations for (2.2):

$$
\begin{array}{r}
c u_{, t}+G_{1} v_{, t}+G_{2, u}=0 \\
b u_{, t}+c u_{, x}-G_{1} v_{, x}+G_{3, u}=0
\end{array}
$$

where $G_{2}$ and $G_{3}$ are given by (2.65) and (2.66). In the particular case of $a=\tilde{a}=b=\tilde{b}=0$ and $c=\tilde{c}=\frac{1}{2}$ we recover the result for the hyperbolic equations [20]:

$$
\begin{array}{r}
G_{1}= \pm \frac{1}{2} \\
\lambda=-1 \\
G_{2}=F_{1}(u-v) ; \quad G_{3}=F_{2}(u+v) \\
p(u)-q(u)=2 F_{1}^{\prime} F_{2}^{\prime} \\
u_{, x}-v_{, x}+2 F_{2}(u+v)=0 \\
u_{, t}+v_{, t}+2 F_{1}(u-v)=0
\end{array}
$$

where $F_{1}$ and $F_{2}$ are solutions of (2.71).

Therefore we have obtained results for the hyperbolic equations. This means that the results of this subsection concern whole class of the hyperbolic equations covering their particular form of the sine-Gordon type [20], [21]. 
2.3.3 The case: $G_{1}=0, \tilde{a}=0, \tilde{b}=0, \tilde{c}=0$

(Note that $\tilde{a}=\tilde{b}=\tilde{c}=0 \Longleftarrow G_{1}=0$ ). This case is trivial because the second equation of $(2.2)$ does not correspond to any differential equation: $Q(v)=0$.

\section{Dual equations for coupled second order PDE in $1+1$ di- mensions}

Let us consider the following set of two coupled PDE-s:

$$
\begin{aligned}
a u_{, x x}+b u_{, t t}+2 c u_{, x t} & =P(u, v) \\
\tilde{a} v_{, x x}+\tilde{b} v_{, t t}+2 \tilde{c} v_{, x t} & =Q(u, v)
\end{aligned}
$$

where $u(x, t)$ and $v(x, t)$ satisfy $(2.3), P(u, v) \in L^{1}(\mathbf{R} \times \mathbf{R})$ and $Q(u, v) \in L^{1}(\mathbf{R} \times \mathbf{R})$. Additionally we assume that $V_{, u}=P(u, v), V_{, v}=Q(u, v)$. The equation parameters $a, b, c, \tilde{a}, \tilde{b}, \tilde{c}$ are arbitrary real constants. All considerations run parallel to Section 2. Therefore the action functional takes the following form:

$$
\begin{gathered}
\Phi[u, v]=\int_{E^{2}}\left[\frac{a}{2} u_{, x}^{2}+\frac{b}{2} u_{, t}^{2}+c u_{, x} u_{, t}+\right. \\
\left.\left.\frac{\tilde{a}}{2} v_{, x}^{2}+\frac{\tilde{b}}{2} v_{, t}^{2}+\tilde{c} v_{, x} v_{, t}+V(u, v)\right)\right] d x d t
\end{gathered}
$$

Using the concept of the strong necessary conditions we derive the following field equations:

$$
\begin{gathered}
u: \quad P(u, v)+G_{1, u}\left(u_{, x} v_{, t}-u_{, t} v_{, x}\right)+G_{2, u u} u_{, x}+G_{2, u v} v_{, x}+G_{3, u u} u_{, t}+G_{3, u v} v_{, t}=0 \\
v: \quad Q(u, v)+G_{1, v}\left(u_{, x} v_{, t}-u_{, t} v_{, x}\right)+G_{2, u v} u_{, x}+G_{2, v v} v_{, x}+G_{3, v u} u_{, t}+G_{3, v v} v_{, t}=0
\end{gathered}
$$

The equations (3.3)-(3.8) have to be self-consistent. Again, we have six simultaneous equations for the five unknown functions: $u, v, G_{1}, G_{2}, G_{3}$. The system (3.3)-(3.8) becomes the Bogomolny equations if there exists such an Ansatz for $G_{1}, G_{2}, G_{3}$ for which the above equations reduce to two equations for $u$ and $v[20],[16]$. Like in Section 2 the reduction of the number of independent equations by an appropriate choice of $G_{1}, G_{2}, G_{3}$ plays essential role in this procedure. Only for a very special $V(u, v)$ such an Ansatz exists [29]. In most cases of $V(u, v)$ the system (3.3)-(3.8) cannot be reduced to the Bogomolny equations. The (3.3), (3.4) and (3.5)-(3.8) require different ways of treatment. In order to make all 
(3.3), (3.4) and (3.5)-(3.8) self-consistent we reduce (3.3), (3.4) to a tautology and make (3.5)-(3.8) linear dependent. The condition for the reduction of the (3.5)-(3.8) is:

$$
\operatorname{rank}\left[\begin{array}{ccccc}
a & c & 0 & G_{1} & G_{2, u} \\
c & b & -G_{1} & 0 & G_{3, u} \\
0 & -G_{1} & \tilde{a} & \tilde{c} & G_{2, v} \\
G_{1} & 0 & \tilde{c} & \tilde{b} & G_{3, v}
\end{array}\right]=2
$$

\subsection{The Bogomolny equations}

In the limit case of $V(u, v)=p(u)+\lambda q(v)$ the (3.1) becomes (2.2) for $\lambda=1$. Therefore, all considerations for the coupled equations run as in Section 2. However the fixed $\lambda$ value causes additional conditions for the equation parameters.

\subsubsection{The class: $G_{1} \neq 0, a \neq 0, \tilde{a} \neq 0$}

I. Transforming the matrix of equations (3.5)-(3.8) to the Gauss-Jordan form we satisfy (3.9) by setting the following relations for the equation parameters and $G_{1}$ :

$$
\begin{aligned}
\tilde{c} & =c \frac{\tilde{a}}{a} \\
\tilde{b} & =b \frac{\tilde{a}}{a} \\
G_{1}^{2} & =\frac{\tilde{a}}{a}\left(a b-c^{2}\right) \\
a b & >c^{2} \wedge a \tilde{a}>0 \quad \vee \quad a b<c^{2} \wedge a \tilde{a}<0
\end{aligned}
$$

and

II. the following set of differential equations for $G_{2}$ and $G_{3}$ :

$$
\begin{aligned}
\left(a b-c^{2}\right) G_{2, v}+G_{1}\left(a G_{3, u}-c G_{2, u}\right) & =0 \\
a G_{3, v}-G_{1} G_{2, u}-c G_{2, v} & =0
\end{aligned}
$$

It results from (3.11) that $G_{1}=$ const.

III. Analogously to the considerations in 2.3 .1 we derive the condition which reduces the (3.3), (3.4) to tautology:

$$
a G_{3, u}^{2}+b G_{2, u}^{2}-2 c G_{2, u} G_{3, u}-2\left(\left(a b-c^{2}\right) V(u, v)=C^{(3)}\right.
$$

where $C^{(3)} \in \mathbf{R}$.

Since, (3.13), (3.14) are equivalent to (2.34), (2.35) we apply the solutions for $G_{1}$ and $G_{2}$ given by (2.50), (2.51).Substituting $G_{2}$ and $G_{3}$ to (3.15) we derive the following form for the integrable potential $V(u, v)$ ( in the Bogomolny decomposition sense):

$$
V(u, v)=\frac{\alpha}{b} \mathrm{D}\left(F_{1}\right)(\xi) \mathrm{D}\left(F_{2}\right)(\zeta)
$$


where $F_{2}$ and $F_{3}$ are arbitrary functions of $C^{2}$,

$$
\begin{array}{r}
\xi=\frac{1}{2} \frac{-v+\alpha u}{\alpha}, \\
\zeta=v+\alpha u, \\
\alpha=\sqrt{-\frac{a b-c^{2}}{G_{1}{ }^{2}} .}
\end{array}
$$

IV. Therefore if there exist $G_{1}(u, v), G_{2}(u, v)$ and $G_{3}(u, v)$ obeying the (3.13)-(3.15) then for (3.1) with coefficients satisfying (3.10) the (3.5) and (3.6) become the Bogomolny equations:

$$
\begin{aligned}
& a u_{, x}+c u_{, t}+G_{1} v_{, t}+G_{2, u}=0 \\
& b u_{, t}+c u_{, x}-G_{1} v_{, x}+G_{3, u}=0
\end{aligned}
$$

In comparison to the decoupled systems we obtain denumerable class of integrable potentials $V(u, v)$. Which means that each smooth enough $F_{1}$ and $F_{2}$ generate $V(u, v)$ leading to the system which is integrable in the sense of the Bogomolny decomposition. In the particular case of $a=\tilde{a}=b=\tilde{b}=1$ and $c=\tilde{c}=0$ we derive the known result for the non-linear wave equations:

$$
\begin{array}{r}
G_{1}={ }_{-}^{+} \\
G_{3, u}=-G_{2, v} \\
G_{3, v}=G_{2, u} \\
V(u, v)=\frac{1}{2}\left(G_{2, u}^{2}+G_{2, v}^{2}\right) \\
u_{, x}+v_{, t}+G_{2, u}=0 \\
u_{, t}-v_{, x}+G_{3, u}=0
\end{array}
$$

\subsubsection{The class: $G_{1} \neq 0, a=0, \tilde{a}=0$}

Note that $\tilde{a}=0$ results from $a=0$.

I. Analogously to the results of the previous subsection we obtain the equations for the equation parameters and $G_{1}$ :

$$
\begin{aligned}
& \tilde{c} b=c \tilde{b} \\
& G_{1}^{2}=-c \tilde{c} \\
& c \tilde{c}<0
\end{aligned}
$$

II. The following set of the differential equations for $G_{2}$ and $G_{3}$ is:

$$
\begin{aligned}
c G_{2, v}+G_{1} G_{2, u} & =0 \\
c^{2} G_{3, v}-c G_{1} G_{3, u}+b G_{1} G_{2, u} & =0
\end{aligned}
$$

and

III. the following condition reduces $(3.3),(3.4)$ to tautology:

$$
\frac{b}{2} G_{2, u}^{2}-c G_{2, u} G_{3, u}+c^{2} V(u, v)=C^{(4)}
$$


where $C^{(4)} \in \mathbf{R}$.

Since, (3.23), (3.24) are equivalent to (2.62), (2.63) we apply (2.65), (2.66) for further considerations. Substituting formulae for $G_{2}$ and $G_{3}$ to (3.15) we derive the following form for the integrable potential $V(u, v)$ ( in the Bogomolny decomposition sense):

$$
V(u, v)=-\frac{c}{G_{1}^{2}} \mathrm{D}\left(F_{1}\right)\left(\frac{v G_{1}-c u}{G_{1}}\right) \mathrm{D}\left(F_{2}\right)\left(\frac{v G_{1}+c u}{G_{1}}\right)
$$

IV. And the Bogomolny equations for (3.1):

$$
\begin{aligned}
c u_{, t}+G_{1} v_{, t}+G_{2, u} & =0 \\
b u_{, t}+c u_{, x}-G_{1} v_{, x}+G_{3, u} & =0
\end{aligned}
$$

we obtain denumerable class of integrable potentials $V(u, v)$. Which means that each smooth enough $F_{1}$ and $F_{2}$ generate $V(u, v)$ leading to the system which is integrable in the sense of the Bogomolny decomposition. In the particular case of $a=\tilde{a}=b=\tilde{b}=0$ and $c=\tilde{c}=\frac{1}{2}$ we recover the result for the hyperbolic equations [29]:

$$
\begin{array}{r}
G_{1}= \pm \frac{1}{2} \\
G_{2, v}+G_{2, u}=0 \\
G_{3, v}-G_{3, u}=0 \\
V(u, v)=2 G_{2, u} G_{3, u} \\
u_{, x}-v_{, x}+2 G_{3, u}=0 \\
u_{, t}+v_{, t}+2 G_{2, u}=0
\end{array}
$$

\subsubsection{The class: $G_{1}=0, \tilde{a}=0, \tilde{b}=0, \tilde{c}=0$}

This case is trivial because it excludes from considerations the second equation of (3.1), which means no Bogomolny decomposition.

\section{Semi-strong necessary condition concept and the Lax hi- erarchy fifth-order KdV equation}

In order to make our formalism more universal we have to extend the strong necessary condition concept to a semi-strong one [16]. Let $\Phi$ be a functional on a set of differentiable functions. These functions can be regarded as elements of the space $C^{2}$. Let $\Phi$ depend on the higher derivatives of $u(x, t)$ :

$$
\Phi[u]=\int_{t_{1}}^{t_{2}} \int_{X} F\left(u, u_{, t}, u_{, x}, u_{, x x}\right) d x d t
$$

Accordingly, one can investigate the necessary condition for the extremum of (4.1) to exist:

$$
\int_{t_{1}}^{t_{2}} \int_{X}\left(\frac{\partial F}{\partial u} \delta u+\frac{\partial F}{\partial u_{, t}} \delta u_{, t}+\frac{\partial F}{\partial u_{, x}} \delta u_{, x}+\frac{\partial F}{\partial u_{, x x}} \delta u_{, x x}\right) d x d t=0
$$


Now, there are more then one possibilities to satisfy (4.2) beyond the Euler-Lagrange's equation. One of them is a semi-strong necessary condition:

$$
\begin{aligned}
u: \quad F_{, u} & =0 \\
u_{, t}: \quad F_{, u, t} & =0 \\
\frac{\delta}{\delta u_{, x}}: \quad F_{, u, x}-D_{x} F_{, u_{, x x}} & =0 .
\end{aligned}
$$

If (4.1) depends on the higher derivatives of $u(x, t)$ until $u(x, t)_{, k x}$ then (4.5) takes the following extended form:

$$
F_{, u, x}-D_{x} F_{, u, x x}+D_{x}^{2} F_{, u, x x x}-D_{x}^{3} F_{, u, x x x x}+\ldots+(-1)^{k-1} D_{x}^{k-1} F_{, u, k x}=0
$$

where $u_{, k x}$ means the derivative of the order $k$. Semi-strong necessary condition concept supplies a helpful tool for the theory of non-linear partial differential equations.

\subsection{The fifth-order $\mathrm{KdV}$ equation}

We present its application to the Lax hierarchy of the fifth-order KdV equation

$$
u_{, t}+30 u^{2} u_{, x}-20 u_{, x} u_{, x x}-10 u u_{, x x x}+u_{, 5 x}=0
$$

The topology associated with (4.7)is equivalent to that associated with the (2.1). Since

(4.7) is Lagrangean-less equation we transform the field functions $u \longrightarrow \bar{u}_{, x}$

$$
\bar{u}_{, x t}+30 \bar{u}_{, x}^{2} \bar{u}_{, x x}-20 \bar{u}_{, x x} \bar{u}_{, x x x}-10 \bar{u}_{, x} \bar{u}_{, 4 x}+\bar{u}_{, 6 x}=0
$$

This equation possesses the following density of the Lagrangean functional

$$
\mathcal{L}=-\bar{u}_{, x} \bar{u}_{, t}-5 \bar{u}_{, x}^{4}-10 \bar{u}_{, x} \bar{u}_{, x x}^{2}-\bar{u}_{, x x x}^{2}
$$

For further considerations we omit bars $\bar{u} \longrightarrow u$. Therefore, we have to consider two independent fields $u$ and $v$ governed by:

$$
\begin{array}{r}
u_{, x t}+30 u_{, x}^{2} u_{, x x}-20 u_{, x x} u_{, x x x}-10 u_{, x} u_{, 4 x}+u_{, 6 x}=0 \\
v_{, x t}+30 v_{, x}^{2} v_{, x x}-20 v_{, x x} v_{, x x x}-10 v_{, x} v_{, 4 x}+v_{, 6 x}=0
\end{array}
$$

The action functional generating (4.10) takes the following form

$$
\begin{array}{r}
\Phi[u, v]=\int_{E^{2}}\left[u{ }_{, x} u_{, t}+5 u_{, x}^{4}+10 u_{, x} u_{, x x}^{2}+u_{, x x x}^{2}\right. \\
\left.+\lambda_{0}\left(v_{, x} v_{, t}+5 v_{, x}^{4}+10 v_{, x} v_{, x x}^{2}+v_{, x x x}^{2}\right)\right] d x d t
\end{array}
$$

\subsection{The Topological invariants}

According to the Theorem 1 we complete the set of independent invariants with the functionals (2.11), (2.13) and

$$
I_{2}=\int_{E^{2}} D_{x} G_{2}\left(u, v, u_{, x}, v_{, x}, u_{, x x}, v_{, x x}, \ldots, u_{, 5 x}, v_{, 5 x}\right) d x d t
$$




\subsection{The semi-strong necessary conditions for the fifth-order KdV equa- tion}

Since (2.11), (2.13) and (4.12) establish the complete set of the topological invariants we are able to create the following gauge transformed functional:

$$
\Phi^{*}[u, v]=\Phi[u, v]-I_{1}-I_{2}-I_{3}
$$

Corresponding set of the semi-strong necessary conditions for the system (4.10) reads:

$$
\begin{array}{ll}
u: \quad & \frac{\partial \mathcal{L}^{*}}{\partial u}=0 \\
v: \quad & \frac{\partial \mathcal{L}^{*}}{\partial v}=0 \\
\frac{\delta}{\delta u_{, x}}: & \quad \frac{\partial \mathcal{L}^{*}}{\partial u_{, x}}-D_{, x} \frac{\partial \mathcal{L}^{*}}{\partial u_{, x x}}+D_{, x}^{2} \frac{\partial \mathcal{L}^{*}}{\partial u_{, x x x}}-D_{, x}^{3} \frac{\partial \mathcal{L}^{*}}{\partial u_{, 4 x}}+D_{, x}^{4} \frac{\partial \mathcal{L}^{*}}{\partial u_{, 5 x}}=0 \\
\frac{\delta}{\delta v_{, x}}: \quad & \frac{\partial \mathcal{L}^{*}}{\partial v_{, x}}-D_{, x} \frac{\partial \mathcal{L}^{*}}{\partial v_{, x x}}+D_{, x}^{2} \frac{\partial \mathcal{L}^{*}}{\partial v_{, x x x}}-D_{, x}^{3} \frac{\partial \mathcal{L}^{*}}{\partial v_{, 4 x}}+D_{, x}^{4} \frac{\partial \mathcal{L}^{*}}{\partial v_{, 5 x}}=0 \\
u_{, t}: \quad & \frac{\partial \mathcal{L}^{*}}{\partial u_{, t}}=0 \\
v_{, t}: & \frac{\partial \mathcal{L}^{*}}{\partial v_{, t}}=0
\end{array}
$$

where $\mathcal{L}^{*}$ denotes the functional density of (4.13). The explicit form of these conditions is:

$$
\begin{array}{ll}
u: & G_{1, u}\left(u_{, x} v_{, t}-u_{, t} v_{, x}\right)+D_{x} G_{2, u}+D_{t} G_{3, u}=0 \\
v: & G_{1, v}\left(u_{, x} v_{, t}-u_{, t} v_{, x}\right)+D_{x} G_{2, v}+D_{t} G_{3, v}=0 \\
& \frac{\delta}{\delta u_{, x}}: \quad u_{, t}+20 u_{, x}^{3}-10 u_{, x x}^{2}-20 u_{, x} u_{, x x x}+2 u_{, 5 x}=G_{1} v_{, t}+G_{2, u} \\
\frac{\delta}{\delta v_{, x}}: & \lambda_{0}\left(v_{, t}+20 v_{, x}^{3}-10 v_{, x x}^{2}-20 v_{, x} v_{, x x x}+2 v_{, 5 x}\right)=-G_{1} u_{, t}+G_{2, v} \\
& \\
u_{, t}: & u_{, x}=-G_{1} v_{, x}+G_{3, u} \\
v_{, t}: & \lambda_{0} v_{, x}=G_{1} u_{, x}+G_{3, v}
\end{array}
$$

According to the general rules described in the subsection 2.2 we set the following problem. For which forms of $G_{1}, G_{2}, G_{3}$ and $\lambda_{0}$ the set (4.14) $\div$ (4.19) reduces to the Bäcklund transformations?. In the first step we reduce (4.18) and (4.19) to the one equation by making them linear dependent by setting:

$$
G_{1}=1, \quad \lambda_{0}=-1
$$


and

$$
G_{3}(u, v)=\frac{1}{6}(u-v)^{3}+E(u-v)+D
$$

where $D$ and $E$ are arbitrary real constants.

The following Ansatz for $G_{2}$ :

$$
\begin{aligned}
G_{2}\left(u, v, \ldots, u_{, 5 x}, v_{, 5 x}\right)= & (u-v)\left[10\left(u_{, x}^{3}+v_{, x}^{3}\right)-5\left(u_{, x x}^{2}+v_{, x x}^{2}\right)\right. \\
& \left.-10\left(u_{, x} u_{, x x x}+v_{, x} v_{, x x x}\right)+(u+v)_{, 5 x}\right]
\end{aligned}
$$

and (4.20),(4.21) reduce (4.18),(4.19) to the first Bäcklund relation:

$$
u_{, x}+v_{, x}=\frac{1}{2}(u-v)^{2}+D
$$

and (4.16), (4.17) to the second Bäcklund relation:

$$
u_{, t}-v_{, t}=-10\left(u_{, x}^{3}-v_{, x}^{3}\right)+5\left(u_{, x x}^{2}-v_{, x x}^{2}\right)+10\left(u_{, x} u_{, x x x}-v_{, x} v_{, x x x}\right)-u_{, 5 x}+v_{, 5 x}
$$

We have to show that (4.14) and (4.15) become a tautology or they are equivalent to linear combination of (4.23) and (4.24).

Theorem 4. If $G_{1}, G_{2}, G_{3}$ and $\lambda_{0}$ have the form of (4.20),(4.21) and (4.22) then (4.14), (4.15) are equivalent to the Bäcklund relations.

Proof. Let us consider (4.14). Taking into account (4.20) the (4.14) reduces to:

$$
D_{x} G_{2, u}+D_{t} G_{3, u}=0
$$

According to (4.21) the second term of this relation takes the following form:

$$
D_{t} G_{3, u}=(u-v)\left(u_{, t}-v_{, t}\right)
$$

whereas, the first one needs the separate considerations:

$$
G_{2, u}=10\left(u_{, x}^{3}+v_{, x}^{3}\right)-5\left(u_{, x x}^{2}+v_{, x x}^{2}\right)-10\left(u_{, x} u_{, x x x}+v_{, x} v_{, x x x}\right)+u_{, 5 x}+v_{, 5 x}
$$

For further considerations we need the four first derivatives of the (4.23):

$$
\begin{aligned}
u_{, x}+v_{, x} & =\frac{1}{2}(u-v)^{2}+D \\
\left(u_{, x}+v_{, x}\right)_{, x} & \left.=(u-v)\left(u_{, x}-v_{, x}\right)\right) \\
\left(u_{, x}+v_{, x}\right)_{, x x} & \left.=\left(u_{, x}-v_{, x}\right)^{2}+(u-v)\left(u_{, x x}-v_{, x x}\right)\right) \\
\left(u_{, x}+v_{, x}\right)_{, x x x} & \left.=3\left(u_{, x}-v_{, x}\right)\left(u_{, x x}-v_{, x x}\right)+(u-v)\left(u_{, x x x}-v_{, x x x}\right)\right) \\
\left(u_{, x}+v_{, x}\right)_{, 4 x} & =3\left(u_{, x x}-v_{, x x}\right)^{2}+4\left(u_{, x}-v_{, x}\right)\left(u_{, x x x}-v_{, x x x}\right)+(u-v)\left(u_{, 4 x}-v_{, 4 x}\right)
\end{aligned}
$$

Using $(4.28) \div(4.31)$ we reduce expression for $D_{x} G_{2, u}$ to the following form:

$$
\begin{aligned}
& D_{x} G_{2, u}=(u-v)\left[15\left(u_{, x}^{2}+v_{, x}^{2}\right)\left(u_{, x}-v_{, x}\right)-10 u_{, x} u_{, x x x}\right. \\
& \left.\quad+10 v_{, x} v_{, x x x}-5\left(u_{, x}-v_{, x}\right)\left(u_{, x x x}+v_{, x x x}\right)+u_{, 5 x}-v_{, 5 x}\right]
\end{aligned}
$$


Assuming $(4.28) \div(4.31)$ we prove the following relation:

$$
\begin{array}{r}
15\left(u_{, x}^{2}+v_{, x}^{2}\right)\left(u_{, x}-v_{, x}\right)-5\left(u_{, x}-v_{, x}\right)\left(u_{, x x x}+v_{, x x x}\right) \\
=10\left(u_{, x}^{3}-v_{, x}^{3}\right)-5\left(u_{, x x}^{2}-v_{, x x}^{2}\right)
\end{array}
$$

Therefore, $D_{x} G_{2, u}$ takes the following form:

$$
\begin{array}{r}
D_{x} G_{2, u}=(u-v)\left[10\left(u_{, x}^{3}-v_{, x}^{3}\right)\right. \\
\left.-5\left(u_{, x x}^{2}-v_{, x x}^{2}\right)-10 u_{, x} u_{, x x x}+10 v_{, x} v_{, x x x}+u_{, 5 x}-v_{, 5 x}\right]
\end{array}
$$

Substituting (4.26) and (4.34) to (4.25) we derive:

$$
\begin{array}{r}
D_{x} G_{2, u}+D_{t} G_{3, u}=(u-v)\left[u_{, t}-v_{, t}+10\left(u_{, x}^{3}-v_{, x}^{3}\right)\right. \\
\left.-5\left(u_{, x x}^{2}-v_{, x x}^{2}\right)-10 u_{, x} u_{, x x x}+10 v_{, x} v_{, x x x}+u_{, 5 x}-v_{, 5 x}\right]=0
\end{array}
$$

Therefore we derive (4.24). By the analogous way we can prove that (4.15) is equivalent to the Bäcklund relations.

Concluding, we have prove that (4.23) and (4.24) establish the auto-Bäcklund transformations for the fifth-order KdV equation.

\section{Conclusions}

We have shown that due to the strong necessary condition concept the derivation of the dual equations for the systems of the PDE-s of the second order with the derivative-less non-linear term can be reduced to an algorithm. The algorithm works both for uncoupled and coupled systems.

The decoupled systems play an important role in the derivation of the Bäcklund transformations for the non-linear PDE-s. It means that in order to derive the Bäcklund transformations for the non-linear partial differential equation we have to complete this with an independent partial differential equation in such a way that the whole system of equations is associated with the non-trivial homotopy group $\pi_{2}\left(S^{2}\right)$. Next, applying the described method we obtain the Bäcklund transformations and the conditions for their existence. The described procedure for coupled equations runs very similar to that for decoupled ones. However, there are significant differences between the uncoupled and coupled systems.

In Table 1 we summarize conditions for the potential of decoupled and coupled PDE's which admit the dual equations. It is easy to understand why it is possible to derive denumerable classes of the coupled equations admitting the Bogomolny decomposition, whereas the decoupled ones admit only few classes of systems possessing the Bäcklund transformations. As we see for both cases the integrability conditions are expressed by two arbitrary functions $F_{1}$ and $F_{2}$. In the case of decoupled system the function variables $u$ and $v$ apearing in must be separated in (2.47) and (2.48) which means tha $u$ can't appear in (2.48) and vice versa. This leads to the strong constrain for $F_{1}$ and $F_{2}(2.56)$, which can be satisfied by very few functions. Whereas, in the coupled case such a constrain does not exist and any smooth enough functions $F_{1}$ and $F_{2}$ correspond to the integrable system in the Bogomoly sense.

In some cases of the nonlinear PDE the strong necessary conditions must be weaken into 
Table 1. Conditions for existence of the Bäcklund transformations and the Bogomolny decomposition.

\begin{tabular}{|c|c|c|c|c|c|c|c|}
\hline \multicolumn{4}{|c|}{$\begin{array}{l}\text { the Bäcklund transformations } \\
\text { (uncoupled equations) }\end{array}$} & \multicolumn{4}{|c|}{$\begin{array}{l}\text { the Bogomolny decomposition } \\
\text { (coupled equations) }\end{array}$} \\
\hline \multicolumn{2}{|c|}{ elliptic } & \multicolumn{2}{|c|}{ hyperbolic } & \multicolumn{2}{|c|}{ elliptic } & \multicolumn{2}{|c|}{ hyperbolic } \\
\hline $\begin{array}{l}\text { conditions } \\
\text { for } \\
P(u), Q(v)\end{array}$ & $\begin{array}{c}\text { constrains } \\
\text { for } \\
F_{1}, F_{2} \\
\end{array}$ & $\begin{array}{l}\text { conditions } \\
\text { for } \\
P(u), Q(v)\end{array}$ & $\begin{array}{c}\text { constrains } \\
\text { for } \\
F_{1}, F_{2} \\
\end{array}$ & $\begin{array}{c}\text { conditions } \\
\text { for } \\
V(u, v)\end{array}$ & $\begin{array}{c}\text { constrains } \\
\text { for } \\
F_{1}, F_{2}\end{array}$ & $\begin{array}{c}\text { conditions } \\
\text { for } \\
V(u, v)\end{array}$ & $\begin{array}{c}\text { constrains } \\
\text { for } \\
F_{1}, F_{2} \\
\end{array}$ \\
\hline$(2.47),(2.48)$ & $(2.56)$ & $(2.69),(2.70)$ & $(2.71)$ & $(3.16)$ & no & $(3.26)$ & no \\
\hline
\end{tabular}

the semi-strong necessary conditions concept. Which is closer to the Euler-Lagrange equations method. For the first time we have applied this procedure to the KdV equation [15]. In this paper we present application of the semi-strong necessary conditions to the fifthorder KdV equation. As the finale result we present the Bäcklund transformations.

The described here algorithm can be easily extended into the systems of $m$ partial differential equations for $p$ unknown functions in an arbitrary $n$-dimensional space of the independent variables. The simplest case occurs if $p=n$. Then we set up boundary conditions for which any continuous solution $u_{1}, u_{2} \ldots, u_{n}$ generates a mapping belonging to the $\pi_{n}\left(S^{n}\right)$ homotopy group. Therefore, we should take into account an optimal number of topological invariants [22]. In the case of $p<n$ we have to supply the investigated system with some independent systems in such a way that the supplemented system will generate the $\pi_{n}\left(S^{n}\right)$ homotopy group. This procedure is a combination of both: the Bäcklund transformation and the Bogomolny decomposition.

\section{Appendix A}

In this Appendix we approach to the simplest case of the Bäcklund transformations [1]- [6]. Suppose we consider two uncoupled partial differential equations, in two independent variables $x$ and $t$, for the two functions $u$ and $v$ :

$$
E q_{1}(u)=0, \quad E q_{2}(v)=0
$$

where $E q_{1}$ and $E q_{2}$ are two operators, which are in general nonlinear. Let $R_{i}=0$ be a pair of relations,

$$
R_{i}\left(u, v, u_{, x}, v_{, x}, u_{, t}, v_{, t}, \cdots ; x, t\right)=0, \quad i=1,2
$$

between the two functions $u$ and $v$. The $R_{i}=0$ is a Bäcklund transformation if it is integrable for $v$ after the $E q_{1}(u)=0$ is fulfilled and if the resulting $v$ is a solution of $E q_{2}(v)=0$, and vice versa. If $E q_{1} \equiv E q_{2}$, so that $u$ and $v$ satisfy the same equation, the $R_{i}$ is called an auto-Bäcklund transformation. This approach to the solution of (6.1) is useful if (6.2) are simpler than (6.1). Usually the order of (6.1) is lower than the order of (6.2). 


\section{Appendix B}

An elementary approach to the Bogomolny equations can be explained with the problem of minimum of the following functional $[8],[30]$ :

$$
H[u]=\int_{-\infty}^{+\infty}\left[\frac{1}{2}\left(\frac{d u(x)}{d x}\right)^{2}+U[u(x)]\right] d x
$$

where $u(x) \in C^{2}(\mathbf{R}), U(u) \in C^{1}(\mathbf{R})$ and $U(u) \in L^{1}(\mathbf{R})$. Then the ground state of (7.1) must satisfy the associated Euler's equation:

$$
y(x)_{, x x}=U_{, y}[y(x)] .
$$

Following the Bogomolny decomposition one splits $H[y]$ :

$$
H[y]=\frac{1}{2} \int_{-\infty}^{+\infty}\left[\frac{d y(x)}{d x} \pm \sqrt{2(U[y]-C)}\right]^{2} d x+I_{0}
$$

where

$$
I_{0}=\overline{+} \int_{-\infty}^{+\infty} \frac{d y(x)}{d x} \sqrt{2(U[y]-C)} d x+\int_{X} C d x
$$

is a topological invariant. $C$ is a constant satisfying the following condition:

$$
\left|\int_{-\infty}^{+\infty} C d x\right|<\infty
$$

It results from (7.3) that, $y(x)$ is the minimum of (7.1) if and only if $y(x)$ satisfies the first order differential equation:

$$
\frac{d y(x)}{d x} \pm \sqrt{2(U[y]-C)}=0
$$

The (7.6) is called the Bogomolny equation.

\section{Acknowledgment}

One of us (T.W.) wish to express many thanks to Ms. Barbara Gwóźdź for strong inspirations.

\section{References}

[1] Wadati M, Sanuki H and Konno K, Relationships among inverse method, Bäcklund transformations and an infinite number of conservation laws, Progr. Theoret. Phys. 53 (1975), 419-436.

[2] Brandt F, Bäcklund transformations and zero-curvature representations of systems of partial differential equations, hep-th/9405064 v1.

[3] Fokas A S, Symmetries and integrability, Stud. Appl. Math. 77 (1987), 253-299. 
[4] Rogers C, Bäcklund transformations in soliton theory, in soliton theory: A survey of results, Editor: Fordy A P, Manchester University Press, Manchester, 1990, 97-130.

[5] Harrison B K, in Lecture Notes in Physics 226, Non-linear equations in classical and quantum field theory, ed. by N. Sanchez, Springer-Verlag, Berlin-Heidelberg-New York-Tokyo (1985), p. 45-56.

[6] Rund H, Variational problems and Bäcklund transformations associated with the sineGordon and Korteweg-de Vries equations and their extantions, in Lectures Notes in Mathematics 515, Bäcklund Transformations, the Inverse Scattering Method, Solitons, and their Applications, Editor: R. M. Miura, Springer-Verlag, Berlin Heidelberg-New York, 1976, 199-226.

[7] Cieśliński J, An algebraic method to construct the Darboux matrix, J. Math. Phys. 36 (1995), 5670-5706.

[8] Bogomolny E B, Stability of classical solutions, Sov. J. Nucl. Phys. 24 (1976), 861870.

[9] Belavin A A, Polyakov A M, Schwartz A S and Tyupkin Yu S, Pseudoparticle solutions of the Yang-Mills equations, Phys. Lett. B 59 (1975), 85-87.

[10] de Vega H J and Schaposnik F A, Classical vortex solution of the Abellian-Higgs model, Phys. Rev. D14 (1976), 1100-1106.

[11] Hong J, Kim Y and Pac P Y, Multivortex solutions of the Abellian-Chern-SimonsHiggs theory, Phys.Rev.Lett. 64 (1990), 2230-2233.

[12] Jackiw R and Weinberg E J, Self-dual Chern-Simons vortices, Phys.Rev. Lett. 64 (1990), 2234-2237.

[13] Olive D and Witten E, Supersymmetry algebra that include topological charges, Phys. Lett. B 78 (1978), 97-101.

[14] P. West, Introduction to Supersymmetry and Supergravity, World Scientific (1986).

[15] Sokalski K, Wietecha T and Lisowski Z, Variational approach to the Bäcklund transformations, Acta Phys. Pol. B32(2001), 17-28.

[16] Sokalski K, Wietecha T and Lisowski Z, Unified variational approach to the Bäcklund transformation and the Bogomolny decomposition, Int. J. Theor. Phys., Group Theor. and Nonlin. Opt., NOVA 9 (2002), 331-354.

[17] Sokalski K, Instantons in anisotropic ferromagnets, Acta Phys. Pol, A56(1979), 571574 .

[18] Sokalski K, Dynamical stability of instantons, Phys. Lett. A 81 (1981), 102-103.

[19] Sokalski K, Instantons in three-dimensional Heisenberg ferromagnets, Acta Phys. Pol. A65 (1984), 457-458. 
[20] Sokalski K, Wietecha T and Lisowski Z, A concept of strong necessary condition in nonlinear field theory, Acta Phys. Pol. B32 (2001), 2771-2791.

[21] Scott A C,Nuovo Cim. B69 (1970) 241.

[22] Wietecha T, Thesis, Jagellonian University, Cracow 2003.

[23] Byrnes S G, Bäcklund transformations and the equation $z_{x y}=F(x, y, z), J$. Math. Phys., 17(1976), 836-842.

[24] Leibbrandt G, Exact solutions of the elliptic sine equation in two space dimensions with applications to the Josphson effect, Phys. Rev. B15 (1977), 3353-3361.

[25] Leibbrandt G, Conservation laws for the elliptic sine equation, Phys. Rev. D16(1977), 970-973.

[26] Leibbrandt G, Solitonlike solutions of the elliptic sine-cosine equation by means of harmonic functions, J. Math. Phys. 19(1978), 960-966.

[27] Borisov A B and Kiseliev V V, Topological defects in incommensurate magnetic and crystal structures and quasi-periodic solutions of the elliptic sine-Gordon equationPhysica, D31(1988) 49-64.

[28] Ferrer R, Elliptic sine-Gordon solitons in Heisenberg plane,Phys. Status Solidi, B199(1997), 535-539.

[29] Sokalski K, Stȩpień Ł and Sokalska D, The existence of Bogomolny decomposition by means of strong necessary conditions, J. Phys. A:Math. Gen. 35 (2002), 6157-6168.

[30] Felsager B, Geometry, particles and fields, Odense University Press, 1981, p. 138.

[31] Drazin P G and Johnson R S, Cambridge Text in Applied Mathematics, Solitons: an introduction, Cambridge University Press, Cambridge, New York, New Rochelle, Melbourne, Sydney, 1989, p. 96. 